\title{
Isothermal Crystallization of Iron-Based Amorphous Alloys in a High Magnetic Field
}

\author{
Reisho Onodera, ${ }^{1, *}$, Shojiro Kimura ${ }^{1}$, Kazuo Watanabe ${ }^{1}$, Yoshihiko Yokoyama ${ }^{2}$, \\ Akihiro Makino $^{2}$ and Keiichi Koyama ${ }^{3}$ \\ ${ }^{1}$ High Field Laboratory for Superconducting Materials, Institute for Materials Research, Tohoku University, Sendai 980-8577, Japan \\ ${ }^{2}$ Advanced Research Center of Metallic Glasses, Institute for Materials Research, Tohoku University, Sendai 980-8577, Japan \\ ${ }^{3}$ Graduate School of Science and Engineering, Kagoshima University, Kagoshima 890-0065, Japan
}

\begin{abstract}
Isothermal crystallization of soft magnetic Fe-Si-B amorphous alloys under high magnetic fields was investigated by magnetization measurements. The increase of magnetization due to the precipitation of ferromagnetic $\alpha$ - $\mathrm{Fe}(\mathrm{Si})$ from the paramagnetic amorphous matrix was observed as a function of time. The magnetic field dependence of the time evolution of the crystallization was also examined. The relaxation time increases under high applied magnetic fields indicating that the suppression of the precipitated crystal growth rate occurs. Moreover, by normalizing the relaxation time of the crystallization, the time evolutions of the crystallized fraction converge to a universal sigmoidal curve regardless of the applied magnetic field strength. These behaviors indicate that the magnetic field influences the growth rate rather than the elementary processes of crystallization. [doi:10.2320/matertrans.M2013136]
\end{abstract}

(Received April 4, 2013; Accepted May 2, 2013; Published June 25, 2013)

Keywords: amorphous alloys, high magnetic field, isothermal crystallization, magnetization measurement

\section{Introduction}

Microstructure control is one of the most important research themes in materials science. It plays an important role in tuning the characteristics of materials, such as their mechanical, electric, and magnetic properties, etc. and has been strenuously investigated for the development of new functional materials. The control of microstructure is often achieved by managing recrystallization and/or atomic diffusion during materials processing. Thermal and chemical treatments are the most fundamental methods of controlling material microstructure. However, recrystallization and diffusion control by thermal treatment or adjustment of chemical composition are hardly sufficient to achieve homogeneous crystal grain size and shape, which are important for material functional properties. Hence, the search for a new crystallization controlled parameter is required to achieve accurate microstructure control for the development of new functional materials.

Many approaches have been examined to improve the functionalities of amorphous alloys intended for structural, biomedical, and magnetic applications ${ }^{1,2)}$ by forming dispersed nanocrystal textures in an amorphous matrix. ${ }^{3-5)}$ The control of crystallization in amorphous alloys is imperative to achieve the desired characteristics. For instance, the grain size and the volume fraction of precipitated crystal phases should be controlled in soft magnetic nanocrystalline alloys in order to obtain good soft magnetic properties such as high saturation magnetization, low coercivity, and high permeability. In recent years, the effects of magnetic fields applied during the annealing of amorphous alloys have been investigated by several groups. The magnetic anisotropy of Fe-Co-Mo-B nanocrystalline alloys induced by magnetic field annealing was investigated by Škorvánek et al. ${ }^{6}$ ) The effect of a high magnetic field on the crystallization of a non-magnetic bulk metallic glass was investigated by Wang

*Graduate Student, Tohoku University et al. ${ }^{7)}$ Fujii et al. reported an enhancement of $\{110\}$ texture formation in $\alpha-\mathrm{Fe}(\mathrm{Si})$ phase of the $\mathrm{Fe}-\mathrm{Si}-\mathrm{B}-\mathrm{Nb}-\mathrm{Cu}$ nanocrystalline alloy and an increase of the nucleation rate of $\alpha-\mathrm{Fe}(\mathrm{Si})$ by annealing in magnetic fields. ${ }^{8}$ These reports indicate the possibility of controlling crystallization and magnetic functionalities by thermal treatment in a magnetic field. Thus, the control of crystallization of amorphous alloys by application of a magnetic field is expected to realize high performance soft magnetic nanocrystalline alloys. The reason is that the magnetic properties of soft magnetic nanocrystalline alloys as typified by FINEMET ${ }^{9)}$ depend strongly on their precipitated crystal grain sizes. ${ }^{10)}$ In the abovementioned reports, field effects on the crystallized texture were mostly analyzed by magnetization measurements, X-ray diffraction, and transmission electron microscopy (TEM) imaging. However, there are very few reports that deal with the crystallization kinetics during isothermal annealing in high magnetic fields. Wolfus et al. investigated isothermal crystallization kinetics for $\mathrm{Fe}_{83} \mathrm{Si}_{5} \mathrm{~B}_{12}$ amorphous alloys using magnetization measurements, although the magnetic field in their measurements was limited to $1 \mathrm{~T},{ }^{11}$ ) and reported an acceleration of the crystallization process. On the other hand, Fujii et al. reported the effect of a magnetic field on the crystallized texture of $\mathrm{Fe}-\mathrm{Si}-\mathrm{B}$ and $\mathrm{Fe}-\mathrm{Si}-\mathrm{B}-\mathrm{Nb}-\mathrm{Cu}$ annealed at $6 \mathrm{~T}^{8,12)}$ These reports suggest that isothermal measurement in higher fields is required, in order to clarify the field effect on the crystallization kinetics. Further, in our recent research, we have observed the crystallization kinetics of $\mathrm{Fe}_{79} \mathrm{Si}_{12} \mathrm{~B}_{9}$ amorphous alloys in high magnetic fields by differential thermal analysis, and revealed a shift of the crystallization peaks toward higher temperature with higher magnetic field. ${ }^{13)}$ We interpreted that this phenomenon originates from the suppression of the crystallization by the magnetic field. The crystallization of this material is accompanied by magnetic transition from the paramagnetic amorphous to the ferromagnetic crystal phase. Thus, one may expect an enhancement of crystallization by applying a magnetic field, because the gain of the Zeeman energy of the 
precipitated ferromagnetic crystal phase is larger than that of the amorphous phase. However, contrary to this expectation, suppression of crystallization was revealed from our experimental results. ${ }^{13)}$ The suppression of the crystal growth leads to the small crystal grain. Hence, this magnetic field effect is important for the development of nanocrystalline alloys with excellent soft magnetic properties.

In order to clarify this magnetic field effect, in this work, isothermal magnetization measurements are carried out on $\mathrm{Fe}_{79} \mathrm{Si}_{12} \mathrm{~B}_{9}$ (Fe-Si-B) amorphous alloys. From these measurements, we obtain the vital information about the kinetics of the crystallization process, including the relaxation time and growth mechanism. The magnetic field dependence of the time evolution of the crystallized fraction is described in detail.

\section{Experimental Procedure}

Fe-Si-B amorphous ribbon with about $23-24 \mu \mathrm{m}$ thickness and about $3 \mathrm{~mm}$ width was prepared by single-roll meltspinning. Magnetization measurements were carried out using a high field vibrating sample magnetometer (VSM) equipped with an electric furnace and a cryocooled superconducting magnet. The sample temperature was measured by using $\mathrm{Pt}-13 \% \mathrm{Rh}$ thermocouple. We confirmed that the influence of a magnetic field on the electromotive force of the thermocouple is negligibly small. The temperature accuracy of the experiments was within $1 \mathrm{~K}$. The temperature $T$ dependence of the magnetization was measured to confirm the Curie temperature $\left(T_{\mathrm{C}}\right)$ and crystallization temperature $\left(T_{\mathrm{x}}\right)$ of the $\mathrm{Fe}-\mathrm{Si}-\mathrm{B}$ amorphous alloy in a temperature range of $300-1100 \mathrm{~K}$. Isothermal magnetization measurements between $T_{\mathrm{C}}$ and $T_{\mathrm{x}}$ were carried out at 713,723 and $733 \mathrm{~K}$ in magnetic fields $B$ of $0.5,5$ and $10 \mathrm{~T}$. In all measurements, the sample was coiled and set in a quartz tube of $8 \mathrm{~mm} / 6 \mathrm{~mm}$ outside/inside diameter and $6 \mathrm{~mm}$ length, and then placed in the measurement probe. The magnetic field was applied along the rotational axis of the coiled ribbon, parallel to the ribbon surface. The sample space was evacuated to less than $10 \mathrm{~Pa}$ by a rotary pump during magnetization measurements.

\section{Results and Discussion}

Figure 1 shows the temperature dependence of the magnetization in $T \leq 1100 \mathrm{~K}$ at $0.5 \mathrm{~T}$. The Curie temperature $T_{\mathrm{C}}$ of the amorphous phase is determined to be $T_{\mathrm{C} \text {,amo. }}=$ $706 \mathrm{~K}^{13)}$ In the temperature region below $T_{\mathrm{x} 1}$, the magnetization decreases as the temperature increases. It then increases abruptly in two steps at crystallization temperatures $T_{\mathrm{x} 1}=758 \mathrm{~K}$ and $T_{\mathrm{x} 2}=808 \mathrm{~K}$, which were determined by DTA measurements. ${ }^{13)}$ The increase of the magnetization indicates the precipitation of ferromagnetic crystals from the paramagnetic amorphous phase. $\alpha$-Fe containing Si crystallizes at the first crystallization temperature, $T_{\mathrm{x} 1}$, and $\mathrm{Fe}_{2} \mathrm{~B}$ crystallizes at the second crystallization temperature, $T_{\mathrm{x} 2 .}{ }^{14}$ ) To obtain nanocrystalline alloys, the process of $\alpha$-Fe precipitation during the first crystallization is important. Therefore, we chose annealing temperatures between $T_{\mathrm{C} \text {,amo }}$. and $T_{\mathrm{x} 1}$ of 713,723 and $733 \mathrm{~K}$ for the isothermal magnetization measurements.

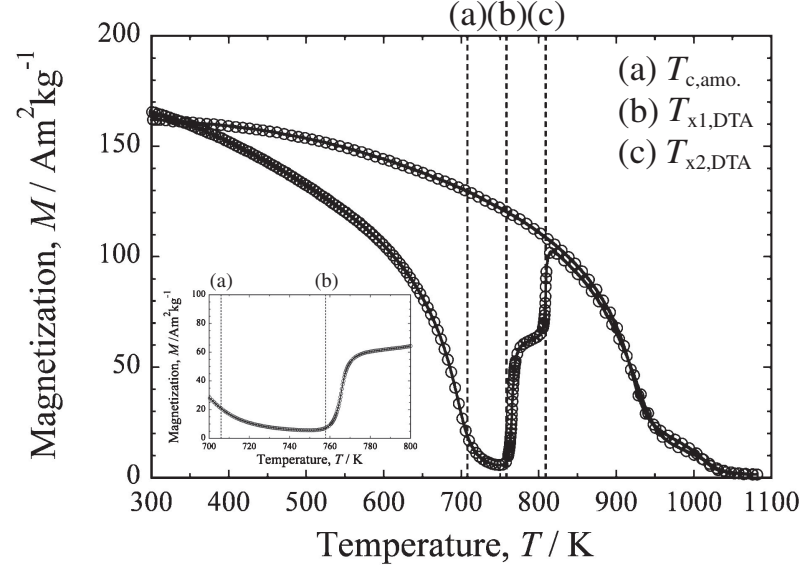

Fig. 1 Temperature dependence of magnetization at $0.5 \mathrm{~T}$. Dashed lines indicate the Curie temperature of the amorphous phase, (a) $T_{\mathrm{C} \text {,amo, }}$, and the

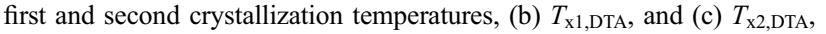
determined by DTA at 0 T. ${ }^{13)}$ Inset shows enlarged view between 700 and $800 \mathrm{~K}$.

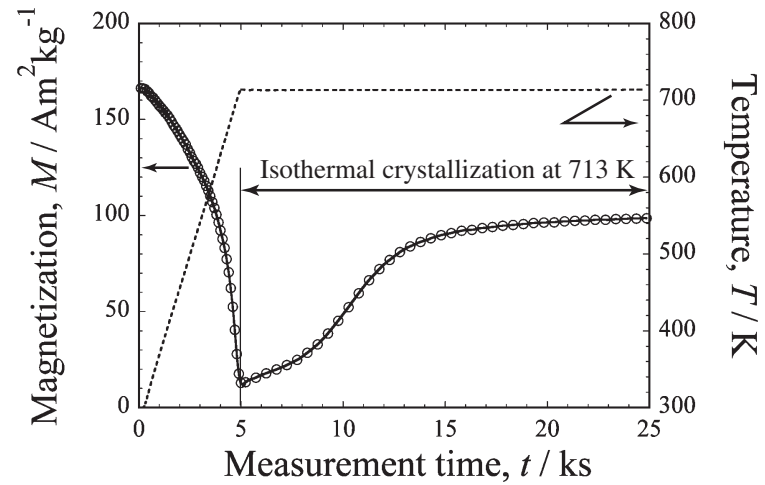

Fig. 2 Time dependence of the sample temperature (dashed line) and the magnetization (open circle) at $0.5 \mathrm{~T}$.

Figure 2 shows the time dependence of magnetization measurements at $0.5 \mathrm{~T}$. At first, the sample is heated to $713 \mathrm{~K}$ from room temperature with constant heating rate $(5 \mathrm{~K} / \mathrm{min})$ in $B=0.5 \mathrm{~T}$. Then, when the sample temperature reaches the annealing temperature, $T_{\mathrm{a}}=713 \mathrm{~K}$, the sample heating is stopped and the sample temperature keeps at $T_{\mathrm{a}}$. The isothermal magnetization shows a sigmoidal increase after the sample heating is stopped, which indicates the precipitation of ferromagnetic $\alpha$-Fe. Therefore, we can obtain the time evolution of the isothermal crystallization of $\alpha$-Fe by analyzing the isothermal magnetization process as discussed below.

Figures 3(a)-3(c) shows the time evolution of the crystallized fraction $x(\tau)$ determined from the increase of magnetization during isothermal annealing in high magnetic fields at $T_{\mathrm{a}}=713,723$ and $733 \mathrm{~K}$, which correspond to 94,95 and $97 \%$ of the first crystallization temperature, $T_{\mathrm{x} 1}=758 \mathrm{~K}$, under zero magnetic field, respectively. The elapsed time $\tau$ of the isothermal process is defined as $\tau=t-t_{\mathrm{s}}$. Here, $t$ is the measurement time and $t_{\mathrm{s}}$ is the time at which the isothermal process starts. The inset of Fig. 3(a) shows the time evolution of the magnetization as a function of $\tau$ during isothermal annealing at $T_{\mathrm{a}}=713 \mathrm{~K}$ and $B=0.5,5$ and $10 \mathrm{~T}$. The crystallized fraction $x(\tau)$ can be estimated from the isothermal magnetization process by the following equation: ${ }^{11}$ 

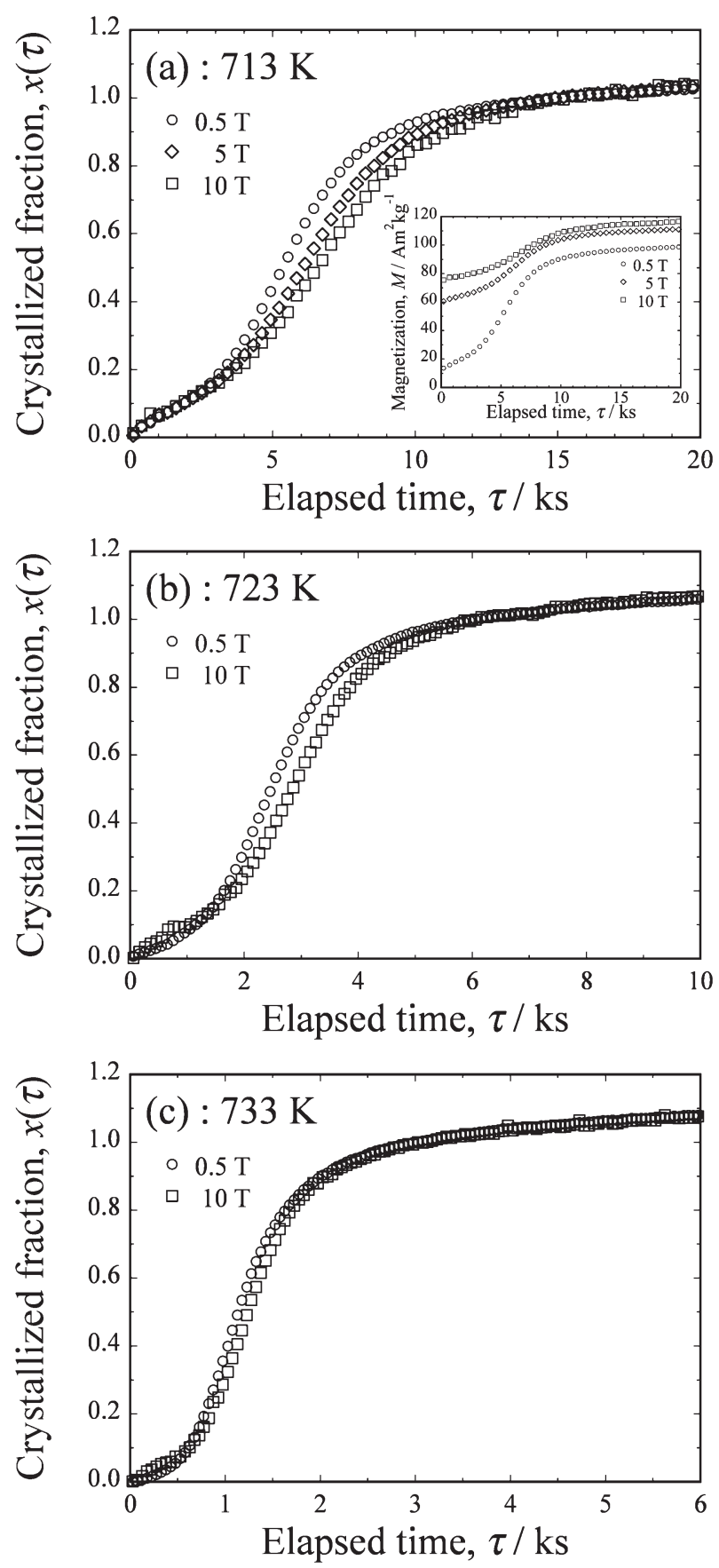

Fig. 3 Time evolution of crystallized fraction in zero and finite magnetic fields at (a) 713, (b) 723 and (c) $733 \mathrm{~K}$. Inset shows magnetization curves as a function of the elapsed time.

$$
x(\tau)=\frac{M(\tau)-M(0)}{M(\infty)-M(0)} .
$$

Here, $M(0)$ is the magnetization at $\tau=0$ and $M(\infty)$ is the magnetization at $\tau_{\infty}=15,6$ and $3 \mathrm{ks}$ for $T_{\mathrm{a}}=713,723$ and $733 \mathrm{~K}$, respectively. The time evolution of the magnetization should saturate when the crystallization of $\alpha$-Fe completes. However, in each measurement, the magnetization still shows a slight increase with time even above $\tau_{\infty}$. This behavior is probably due to the fact that the second crystallization temperature $T_{\mathrm{x} 2}$ is close to $T_{\mathrm{x} 1}$. At this temperature region, the second crystallization has longer relaxation time in comparison with that of the first crystallization. Therefore, it can be

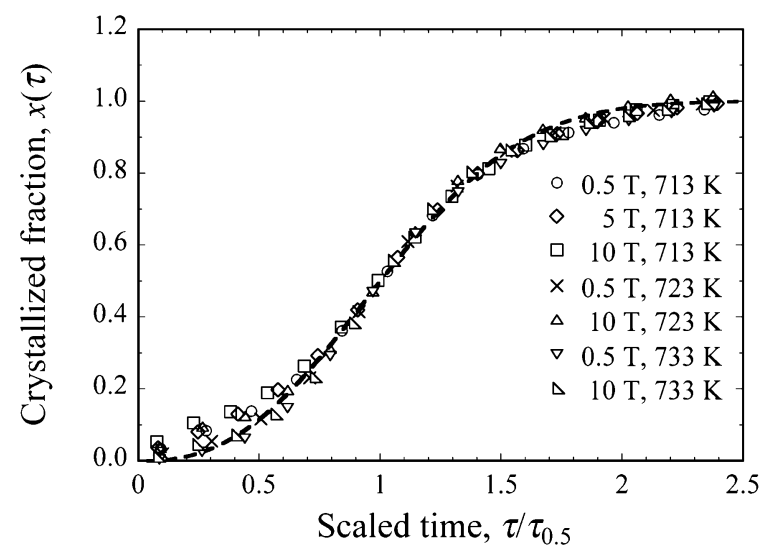

Fig. 4 Crystallized fraction as a function of the scaled time obtained under all experimental conditions. Dashed curve shows the theoretical curve calculated using the scaled Johnson-Mehl-Avrami equation [eq. (3)] with $b=0.69$ and $n=2.5$.

considered that the increase of magnetization due to the second crystallization is overlapped on the final stage of the first crystallization. Such magnetization behaviors were also found in Wolfus's results below $1 \mathrm{~T}^{11)}$

The sigmoidal curves in Fig. 3(a) indicate that the crystal growth becomes more gradual in higher magnetic fields, because the elapsed time, $\tau_{0.5}$, at which the crystallized fraction $x(\tau)$ approaches $x=0.5$, increases with magnetic field strength. The $\tau_{0.5}$ values are estimated to be $\tau_{0.5}=5.3$, 6.1 and $6.6 \mathrm{ks}$, for $B=0.5,5$ and $10 \mathrm{~T}$, respectively. Thus, we found a suppression of the growth rate of the precipitated crystal phase in high magnetic fields. In our previous study, we suggested suppression of crystallization in high magnetic fields by DTA. ${ }^{13)}$ From the present results, it was found that the magnetic field affects the growth process of the crystallization rather than the nucleation. The sigmoidal curve of $x(\tau)$ in Fig. 3(b) $\left[T_{\mathrm{a}}=723 \mathrm{~K}\right]$ is similar to that seen in Fig. 3(a) $\left[T_{\mathrm{a}}=713 \mathrm{~K}\right]$. However, the suppression of the growth rate is smaller than that observed in Fig. 3(a). The effect of the magnetic field on the crystallization process at $T_{\mathrm{a}}=723 \mathrm{~K}$ is weaker, compared with that at $T_{\mathrm{a}}=713 \mathrm{~K}$, because the crystallization reaction becomes more active at a higher temperature and smears out the field effect. At the highest temperature of $T_{\mathrm{a}}=733 \mathrm{~K}$, the suppression of the growth rate by the applied magnetic field is negligibly small even at $10 \mathrm{~T}$, as shown in Fig. 3(c).

Figure 4 shows crystallized fraction as a function of the scaled elapsed time, $\tau / \tau_{0.5}$. The crystallization fractions converge into a universal curve regardless of the strength of the applied magnetic field and the annealing temperature, except for the early stage of the crystallization process $(x<0.2)$, at which slight disagreements among the data at different magnetic fields are observed. These behaviors demonstrate that the magnetic field causes suppression of the crystal growth rate but the elementary crystallization process remains unchanged, because if the magnetic field influences the elementary process, the time scaled crystallization fractions under the different magnetic fields would not converge into a universal curve. Generally, the time evolution of crystal growth can be analyzed by the JohnsonMehl-Avrami (JMA) equation: ${ }^{15}$ ) 


$$
x(\tau)=1-\exp \left[-k\left(\tau-\tau_{0}\right)^{n}\right],
$$

where $k$ is the reaction rate constant, $\tau$ is the elapsed time of the isothermal annealing, and $\tau_{0}$ is the incubation time of crystallization. $n$ is the Avrami exponent that depends on the crystallization mechanism. Incubation time $\tau_{0}$ was not observed in the present results. Thus, we assume $\tau_{0}=0$. As shown in Fig. 4, the experimental growth curve can be fitted by the scaled JMA equation: ${ }^{16}$

$$
x\left(\tau / \tau_{0.5}\right)=1-\exp \left[-b\left(\tau / \tau_{0.5}\right)^{n}\right],
$$

calculated with $b=0.69$ and $n=2.5$. Although a slight deviation appears in the region $x<0.2$, the overall behavior of the experimental results agrees with the calculated curve. The Avrami exponent $n \sim 2.5$ of the first crystallization reaction in $\mathrm{Fe}_{78} \mathrm{Si}_{9} \mathrm{~B}_{13}$ has been reported by Jakubczyk et al. ${ }^{17)}$ Cui et al. reported that the local Avrami exponent $n$ of the first crystallization reaction in $\mathrm{Fe}_{84} \mathrm{Si}_{4} \mathrm{~B}_{12}{ }^{18,19)}$ changes from $n>3$ to $n \sim 2.5$ as a function of the crystallization fraction $x(\tau)$. These results are consistent with our result.

An Avrami exponent of $n=2.5$ indicates that the crystallization proceeds with homogeneous nucleation and diffusion-controlled three-dimensional growth. In this case, the growth rate of the precipitated crystal grains is dominated by atomic diffusion. From the isothermal magnetization measurements, we found that the growth rate was suppressed by application of a magnetic field. Therefore, it is suggested that the atomic diffusion on the crystallization process in $\mathrm{Fe}_{79} \mathrm{Si}_{12} \mathrm{~B}_{9}$ is suppressed by an external magnetic field.

\section{Conclusion}

The isothermal crystallization of iron-based amorphous alloy in high magnetic fields has been investigated by magnetization measurements in fields up to $10 \mathrm{~T}$. The time evolutions of the crystallized fraction were estimated from the increase of the magnetization during isothermal annealing. The growth curve becomes more gradual in higher magnetic fields, compared with that at $0.5 \mathrm{~T}$ at each annealing temperature. These behaviors suggest a suppression of the growth rate at high magnetic fields. By scaling the elapsed time of the crystallization, the evolution of the crystallized fraction converges to a universal sigmoidal curve regardless of the annealing temperature and strength of the applied magnetic field. This sigmoidal curve can be fitted by the JMA equation with $b=0.69$ and $n=2.5$, indicating that the elementary crystallization process does not change by applying a magnetic field whereas the growth rate is suppressed. The Avrami exponent of $n=2.5$ indicates diffusion-controlled crystal growth. Therefore, our results suggest that crystallization is suppressed by the applied magnetic field, which inhibits atomic diffusion during the crystallization process.

\section{Acknowledgement}

This work was supported in part by Grants for Excellent Graduate Schools, MEXT, Japan.

\section{REFERENCES}

1) A. Inoue: Acta Metall. 48 (2000) 279-306.

2) F. Qin, X. Wang, S. Zhu, A. Kawashima, K. Asami and A. Inoue: Mater. Trans. 48 (2007) 515-518.

3) C. Fan, A. Takeuchi and A. Inoue: Mater. Trans. JIM 40 (1999) 42-51.

4) Y. Yoshizawa and K. Yamauchi: Mater. Trans. JIM 31 (1990) 307-314.

5) J. Marcin, A. Klinda, P. Švec, D. Praslička, J. Blažek, J. Kováč, P. Švec and I. Škorvánek: IEEE Trans. Magn. 46 (2010) 416-419.

6) I. Škorvánek, J. Marcin, J. Turčanová, J. Kováč and P. Švec: J. Alloy. Compd. 504 (2010) S135-S138.

7) X. D. Wang, M. Qi and S. Yi: Scr. Mater. 51 (2004) 1047-1050.

8) H. Fujii, V. A. Yardley, T. Matsuzaki and S. Tsurekawa: J. Mater. Sci. 43 (2008) 3837-3847.

9) Y. Yoshizawa, S. Oguma and K. Yamauchi: J. Appl. Phys. 64 (1988) 6044-6046.

10) G. Herzer: Scr. Metall. Mater. 33 (1995) 1741-1756.

11) Y. Wolfus, Y. Yeshurun, I. Felner and J. Wolny: Philos. Mag. B 56 (1987) 963-968.

12) H. Fujii, S. Tsurekawa, T. Matsuzaki and T. Watanabe: Philos. Mag. Lett. 86 (2006) 113-122.

13) R. Onodera, S. Kimura, K. Watanabe, S. M. Lee, Y. Yokoyama, A. Makino and K. Koyama: Mater. Trans. 54 (2013) 188-191.

14) Y. Takahara and N. Narita: Mater. Trans. JIM 41 (2000) 1077-1081.

15) W. A. Johnson and R. F. Mehl: Trans. Am. Inst. Min. Metall. Pet. Eng. 135 (1939) 416-442.

16) Y. Takahara: Mater. Trans. JIM 37 (1996) 1453-1457.

17) E. Jakubczyk, A. Krajczyk and M. Jakubczyk: J. Phys. Conf. Series 79 (2007) 012008.

18) L. Cui, H. Men, A. Makino, T. Kubota, K. Yubuta, M. Qui and A. Inoue: Mater. Trans. 50 (2009) 2515-2520.

19) A. Calka and A. P. Radiński: J. Mater. Res. 3 (1988) 59-66. 